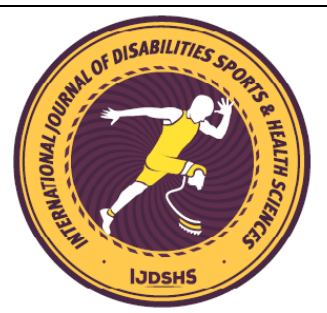

\title{
The Relationship of Kinesiophobia and Pain Catastrophizing with Pain, Range of Motion, Muscle Strength and Function in Osteoarthritis
}

\author{
Ozan GÜR ${ }^{* 1}$ (iD), Selda BAŞAR ${ }^{2}$ (D) Erdinç ESEN $^{3}$ (D), M. Baybars ATAOĞLU ${ }^{4}$ (D), Sacit TURANLI ${ }^{5}$ (iD)
}

\footnotetext{
${ }^{* 1}$ Kirklareli University, Faculty of Health Sciences, Department of Physiotherapy and Rehabilitation, Kirklareli, Turkey

${ }^{2}$ Gazi University, Faculty of Health Sciences, Department of Physiotherapy and Rehabilitation, Ankara, Turkey

${ }^{3,4,5}$ Gazi University, Faculty of Medicine, Department of Orthopedics and Traumatology, Ankara, Turkey

*Corresponding author: ozan.gur94@gmail.com
}

\begin{abstract}
Objective: This study aimed to investigate the relationship between kinesiophobia and pain catastrophizing with pain, range of motion, muscle strength, and function in patients with knee osteoarthritis. Methods: 18 female knee osteoarthritis patients between the ages of 50-70 who were found to have kinesiophobia and pain catastrophizing were included in the study. Kinesiophobia, pain catastrophizing, pain, range of motion, muscle strength, and functional status were evaluated within the scope of the study. Results: A statistically significant strong positive correlation between kinesiophobia and pain intensity during activity $(\mathrm{r}=0.80, \mathrm{p}<0.001)$; a statistically significant moderate negative correlation between kinesiophobia and active knee flexion angle $(\mathrm{r}=-0.48, \mathrm{p}<0.05)$; a statistically significant moderate positive correlation between kinesiophobia and the Five Times Sit to Stand Test time and the Stair Climb Test time $(r=0.51, p<0.05 ; r=0.67, p<0.05$, respectively) was found. A statistically significant moderate positive correlation between pain catastrophizing and resting pain intensity, pain intensity at night, pain intensity during activity, and passive knee extension angle $(r=0.66, p<0.01 ; r=0.61, p<0.01 ; r=0.47, p<0.05 ; r=0.48$, $\mathrm{p}<0.05$, respectively); a statistically significant moderate negative correlation between pain catastrophizing and active knee flexion angle $(\mathrm{r}=-0.49, \mathrm{p}<0.05)$ was found. Conclusions: It was determined that as kinesiophobia increased in patients with knee osteoarthritis, pain, range of motion, muscle strength, and functional status worsened, and the increase in pain catastrophizing was associated with worsening in pain and range of motion. Therefore, it was concluded that reducing kinesiophobia, and pain catastrophizing levels would contribute to improving functions in patients with knee osteoarthritis
\end{abstract}

Keywords

Osteoarthritis, Kinesiophobia, Pain Catastrophizing, Pain, Function

\section{INTRODUCTION}

Osteoarthritis is a degenerative joint disease in which all joint structures are affected, including cartilage, subchondral bone, synovium, ligaments, joint capsule, and muscles. Osteoarthritis, which is the most common form of arthritis, is one of the important health problems that cause severe limitation of movement and pain (Bijlsma et al., 2011; Felson et al., 2000; A. D. Woolf and Pfleger, 2003). Osteoarthritis increases with age and is often seen in the elderly (Loeser Jr, 2000). It is thought that there are around 300 million osteoarthritis patients in the world (James et al., 2018). It has been determined that the prevalence of symptomatic knee osteoarthritis in Turkey is $8 \%$ in men over 50 years old, $22.5 \%$ in women, and $14.8 \%$ in total (Kacar et al., 2005). Osteoarthritis is a disease with multiple etiologies, which is caused by the inflammatory, metabolic and mechanical factors (Felson et al., 2000). Risk factors in knee osteoarthritis are classified as modifiable and non- 
modifiable, such as age, gender, family history, obesity, trauma, and malalignment (Abramoff and Caldera, 2020; Felson et al., 1995; Lespasio et al., 2017; Zhang and Jordan, 2010).

Pain, edema, bone growth, crepitation, locking sensation, stiffness, muscle weakness, decrease in range of motion and deformity are common symptoms in patients with knee osteoarthritis. Knee osteoarthritis negatively affects daily life by causing deterioration in the function and quality of life (Abramoff and Caldera, 2020; Felson et al., 1995; Hunter and Bierma-Zeinstra, 2019).

In long-term pain conditions such as osteoarthritis, sensitivity to pain and chronic pain may develop. This sensitivity and chronic pain occur as a result of neuroplasticity (C. J. Woolf and Salter, 2000). After injuries, while primary hyperalgesia occurs as a result of peripheral sensitization in the site of inflammation; secondary hyperalgesia occurs as a result of central sensitization in areas of the body where there is no inflammation (Coderre et al., 1993). After tissue healing occurs, peripheral and secondary hyperalgesia disappears. However, as in osteoarthritis, if inflammation and pain are prolonged due to continuous anabolic and catabolic activities in the site of injury, sensitization and hypersensitivity continue. This causes an increase in the severity of pain and the formation of chronic pain (Kehlet et al., 2006). Sensitization and hypersensitivity continue in cases of fear developed against pain (Leeuw et al., 2007; Vlaeyen and Linton, 2000). People who react inconsistently and violently to pain develop avoidance behaviors against injury/re-injury. Therefore, kinesiophobia and pain catastrophizing are important psychological factors that cause pain and worsening in function (Leeuw et al., 2007).

Kinesiophobia (Perrot et al., 2018) and pain catastrophizing (M. Sullivan et al., 2009) decrease the efficiency of treatment and patient satisfaction in cases of long-term pain due to their negative effects on pain and function. Therefore, the effects of kinesiophobia and pain catastrophizing in osteoarthritis patients should be determined. For this reason, this study aimed to investigate the relationship between kinesiophobia and pain catastrophizing with pain, range of motion, musle strength and function in knee osteoarthritis patients.

\section{MATERIALS AND METHODS}

\section{Study Design and Participants}

The study was carried out with 18 female knee osteoarthritis patients between the ages of 5070 who were planned to have total knee arthroplasty surgery and were admitted to the Gazi University Hospital Orthopedic Service. Since it was determined that there were differences in muscle morphology and functional outcomes in women compared to men, only female patients were included in the study so that the differences would not affect the results of the study (Behan et al., 2018; Gustavson et al., 2016).

The inclusion criteria of the study were (1) woman between the ages of 50-70, (2) knee osteoarthritis of stage 3-4 according to the Kellgren-Lawrence Osteoarthritis Classification System, (3) Tampa Scale of Kinesiophobia score of $\geq 37$, (4) Pain Catastrophizing Scale score of $\geq 30$. The exclusion criteria of the study were (1) neurological, rheumatological, or oncological disease, (2) anxiety diagnosed by a specialist physician, (3) Mini Mental Test score of <24. The study protocol was approved by the ethics committee of Gazi University (No: 611). All the assessments were conducted in accordance with the Helsinki declaration. All cases provided a written informed consent approved by the ethics committee.

\section{Measurements}

Mini-mental state, kinesiophobia, and pain catastrophizing assessments were performed to determine eligibility for participation in the study. Demographic, physical, and pathological information of the patients were recorded. Also, pain, range of motion, muscle strength, and functional status were evaluated and recorded in the evaluation form.

\section{Mini Mental Test}

The Standardized Mini-Mental Test was used in the evaluation of educated patients, and the Modified Mini-Mental Test was used in the evaluation of illiterate patients (Folstein et al., 1975). The validity and reliability of the Standardized Mini-Mental Test (ICC: 0.99) were performed by Güngen et al. in 2002 (Güngen et al., 2002), and the validity and reliability study of the Modified Mini-Mental Test by Ayhan et al. in 2018 (IC: 0.70) (Ayhan et al., 2018). The MiniMental Tests are methods that allow the numerical evaluation of cognitive status. The tests consist of 
11 items in 5 sections: orientation (time and space orientation), recording memory, attention and calculation, recall, and language. By scoring 11 items, the total score varies between 0-30 (Folstein et al., 1975). The cut-off point of Mini-Mental Test scores was determined as 23/24 (Ayhan et al., 2018; Güngen et al., 2002).

\section{Tampa Scale of Kinesiophobia}

Kinesiophobia was evaluated with the Tampa Scale of Kinesiophobia, of which Turkish validity and reliability were studied (Kori, 1990; Yilmaz et al., 2011). The Tampa Scale of Kinesiophobia includes injury/re-injury and fear of movement parameters and consists of 17 questions. A 4-point Likert scale ( $1=$ strongly disagree, $4=$ totally agree) is used to evaluate the questions. The total score is calculated after reversing the scores of questions $4,8,12$ and 16 . The total score ranges from 17 to 68 (Kori, 1990; Vlaeyen et al., 1995). Scores of 37 and above on the Tampa Scale of Kinesiophobia indicate the presence of high kinesiophobia (ICC: 0.806) (Vlaeyen et al., 1995).

\section{Pain Catastrophizing Scale}

Pain catastrophizing was assessed using the Pain Catastrophizing Scale. In 2017, Ugurlu et al. conducted a Turkish validity and reliability study of the Pain Catastrophizing Scale (Ugurlu et al., 2017). The Pain Catastrophizing Scale is a 13 -item scale that evaluates pain magnification, worrying about pain, and coping with pain. Each item is evaluated on a 5-point Likert scale, between 0-4 points $(0=$ never, $4=$ always). The total score ranges from 0 to 52 points (Domenech et al., 2013; M. J. Sullivan et al., 1995). Scores of 30 and above on the Pain Catastrophizing Scale indicate the presence of high pain catastrophizing (ICC: 0.830) (M. J. Sullivan, 2009).

\section{Numerical Pain Rating Scale}

The pain was evaluated separately with Numerical Pain Rating Scale (NPRS) at rest, at night, and during activity. Pain during activity was evaluated by questioning the pain intensity of the patients on walk throughout the day. The patient was asked to express the severity of pain in numbers that would accurately reflect her pain, with a score of zero if there was no pain, and ten for the most severe pain (ICC: 0.84) (Briggs and Closs, 1999; DeLoach et al., 1998; Jensen and McFarland, 1993).

\section{Range of Motion Testing}

Knee range of motion was measured with a universal goniometer with proven validity and reliability for knee joint flexion and extension movements in the supine position. 2 measurements were made for each evaluation and the highest values were recorded in degrees (Jakobsen et al., 2010; Watkins et al., 1991).

The cases where 0 degrees could not be reached in the evaluation of knee extension were recorded as positive values, and the degrees of knee extension performed more than 0 degrees were recorded as negative values (Jakobsen et al., 2010).

Evaluation of active and passive knee flexion: The pivot point of the goniometer was placed in the middle of the lateral condyle of the femur while the patient was lying in the supine position.

To determine active knee flexion, the patient was asked to flex the knee as much as possible, and the active knee flexion angle was determined so that one arm of the goniometer was parallel to the femur while the other arm followed the midline of the fibula (ICC: 0.81) (Jakobsen et al., 2010).

To determine passive knee flexion, the patient's knee was manually extended without causing pain or discomfort in the patient, and the passive knee flexion angle was determined so that one arm of the goniometer was parallel to the femur while the other arm followed the midline of the fibula (ICC: 0.96) (Jakobsen et al., 2010).

Evaluation of active and passive knee extension: A roller was placed under the heel of the lower extremity to be evaluated while the patient was lying in the supine position, and the pivot point of the goniometer was placed in the middle of the lateral condyle of the femur.

To determine active knee extension, the patient was asked to extend the knee as much as possible, and the active knee extension angle was determined so that one arm of the goniometer was parallel to the femur while the other arm followed the midline of the fibula (ICC: 0.86) (Jakobsen et al., 2010).

To determine passive knee extension, the patient's knee was manually extended without causing pain or discomfort in the patient, and the passive knee extension angle was determined so that one arm of the goniometer was parallel to the femur while the other arm followed the midline of the fibula (ICC: 0.70) (Jakobsen et al., 2010). 


\section{Five Times Sit to Stand Test}

The Five Times Sit to Stand Test (FTSTS) can be used to evaluate lower extremity muscle strength during sitting and standing movements in the elderly (Csuka and McCarty, 1985; Schaubert and Bohannon, 2005; Tiedemann et al., 2008). The patient sat in a standard-height $(43 \mathrm{~cm}$.) chair with their backs against the chair, hands crossed on their chests, and feet touching the floor. During the test, they were asked not to use their hands to get support from the arms or lower extremities of the chair. In addition, participants were allowed to position their feet comfortably. Patients were asked to sit and stand up to 5 times in a row as quickly and safely as they could. FTSTS was repeated one time unless there was a problem in performing the test. When there was a problem in performing the test, the patient was rested and then the test was repeated. The time between the first moment of movement and the moment of resting on a chair for the last time was recorded in seconds with a stopwatch (ICC: 0.80) (Tiedemann et al., 2008).

\section{Timed Up and Go Test}

In the Timed Up and Go Test (TUG), the patient was asked to get up from the chair as fast as she could, walk the specified 3 meters distance, turn around, and sit on the chair by walking the same path again. TUG was repeated one time unless there was a problem in performing the test. When there was a problem in performing the test, the patient was rested and then the test was repeated. The time at which the test was completed was measured with a stopwatch and recorded in seconds (ICC: 0.97) (Bennell et al., 2011; Steffen et al., 2002).

Table 1. Demographic features of the patients

\section{Stair Climb Test}

In the Stair Climb Test (SCT), patients were first asked to climb 9 steps with a step height of approximately $20 \mathrm{~cm}$, then return and descend 9 steps. SCT was repeated one time unless there was a problem in performing the test. When there was a problem in performing the test, the patient was rested and then the test was repeated. The time at which the test was completed was measured with a stopwatch and recorded in seconds (ICC: 0.93) (Bennell et al., 2011; Rejeski et al., 1995).

\section{Statistical Analysis}

SPSS 22.0 statistical package program was used in the analysis of the data. Number (n)percent $(\%)$, mean \pm standard deviation (mean \pm sd), median, and minimum-maximum (min-max) values were used as descriptive statistics. In the study, the conformity of the data to the normal distribution was evaluated with the KolmogorovSmirnov test. Since it was observed that the data were not normally distributed, Spearman correlation analysis was used in the correlation analysis of the data. Spearman's correlation coefficient interpretation is similar to that of Pearsons. The Pearson correlation coefficients were interpreted as; $0-0.19=$ very weak, 0.20 $0.39=$ weak, $0.40-0.69=$ mederate, $0.70-0.89=$ strong, $0.90-1.0=$ very strong (Streiner et al., 2015). Statistical significance level was accepted as $\mathrm{p}<0.05$ in all analyzes performed in the study.

\section{RESULTS}

Age, body mass index, duration of disease, education level, exercise habit and smoking status were evaluated as demographic features of the patients (Table 1).

\begin{tabular}{lcc}
\hline Demographic Features & & $\begin{array}{c}\text { Total } \\
(\mathbf{n}=\mathbf{1 8})\end{array}$ \\
\hline Age (years, mean \pm SD) & $63 \pm 6$ \\
\hline BMI (kg/m2, mean \pm SD) & & $31.2 \pm 4.03$ \\
\hline Duration of Disease [year, med (min-max)] & & $6(2-30)$ \\
\hline Level of Education (n, \%) & & $5(27.8)$ \\
& & $12(66.7)$ \\
\hline Exercise Habit (n, \%) & Uneducated & $1(5.5)$ \\
\cline { 2 - 3 } & Himary School & $0(0)$ \\
\cline { 2 - 3 } Smoking Status (n, \%) & Yes & $18(100)$ \\
\cline { 2 - 3 } & No & $2(11.1)$ \\
\cline { 2 - 3 } & Yes & $16(88.9)$ \\
\hline
\end{tabular}

n: number of patients, SD: standard deviation, med: median, min: minimum, max: maximum, kg: kilogram, m: meter, \%: percentage 
Pain were evaluated at rest, at night and during activity. The median pain intensity of the patients in all pain assessments was found to be 5 or above. In the range of motion measurements, passive and active knee flexion and extension were evaluated. The FTSTS, the TUG and the SCT were the functional tests performed in the study (Table 2).

Table 2. Pain, range of motion, muscle strength, and functional results of the patients

\begin{tabular}{lc}
\hline Variables & $\begin{array}{c}\text { Total } \\
(\mathbf{n}=\mathbf{1 8}) \\
\text { Med (min-max) }\end{array}$ \\
\hline Resting Pain (NPRS) & $5(0-9)$ \\
\hline Night Pain (NPRS) & $6(0-10)$ \\
\hline Activity Pain (NPRS) & $7(2-10)$ \\
\hline Passive Knee Flexion (degree) & $98(20-115)$ \\
\hline Passive Knee Extension (degree) & $1(-5-8)$ \\
\hline Active Knee Flexion (degree) & $81(55-109)$ \\
\hline Active Knee Extension (degree) & $5(0-10)$ \\
\hline FTSTS (second) & $23.85(12.40-51.20)$ \\
\hline TUG (second) & $15.4(8.80-37.90)$ \\
\hline SCT (second) & $30.7(20.60-43.84)$
\end{tabular}

med: median, min: minimum, max: maximum, NPRS: Numerical Pain Rating Scale, FTSTS: Five Times Sit to Stand Test, TUG: Timed Up and Go Test, SCT: Stair Climb Test.

Kinesiophobia was found correlated with pain intensity during activity, active knee flexion angle, the FTSTS time and the TUG time $(\mathrm{p}<0,05)$. There was no statistically significant correlation between kinesiophobia and resting pain intensity, pain intensity at night, active knee extension angle and the SCT $(p>0.05)$. Pain catastrophizing was found correlated with resting pain intensity, pain intensity at night, pain intensity during activity, passive knee extension angle and active knee flexion angle $(\mathrm{p}<0.05)$. There was no statistically significant correlation between pain catastrophizing and functional tests $(\mathrm{p}>0.05)$, (Table 3).

Table 3. Correlation between kinesiophobia and pain catastrophizing with pain, range of motion, muscle strength, and function

\begin{tabular}{lcccc}
\hline & \multicolumn{2}{c}{ TSK } & \multicolumn{1}{c}{ PCS } \\
\cline { 2 - 5 } Resting Pain (NPRS) & $\mathbf{r}$ & $\mathbf{p}$ & $\mathbf{~}$ & $\mathbf{0 . 6 6}$ \\
\hline Night Pain (NPRS) & 0.40 & 0.093 & $\mathbf{0 . 0 0 3}$ \\
\hline Activity Pain (NPRS) & 0.34 & 0.161 & $\mathbf{0 . 6 1}$ & $\mathbf{0 . 0 0 7}$ \\
\hline Passive Knee Flexion (degree) & $\mathbf{0 . 8 0}$ & $\mathbf{0 . 0 0 0}$ & $\mathbf{0 . 4 7}$ & $\mathbf{0 . 0 4 7}$ \\
\hline Passive Knee Extension (degree) & 0.23 & 0.350 & -0.31 & 0.902 \\
\hline Active Knee Flexion (degree) & 0.22 & 0.368 & $\mathbf{0 . 4 8}$ & $\mathbf{0 . 0 4 1}$ \\
\hline Active Knee Extension (degree) & $\mathbf{- 0 . 4 8}$ & $\mathbf{0 . 0 4 4}$ & $\mathbf{- 0 . 4 9}$ & $\mathbf{0 . 0 3 9}$ \\
\hline FTSTS (second) & 0.18 & 0.471 & 0.29 & 0.233 \\
\hline TUG (second) & $\mathbf{0 . 6 7}$ & $\mathbf{0 . 0 2 2}$ & 0.28 & 0.397 \\
\hline SCT (second) & $\mathbf{0 . 5 1}$ & $\mathbf{0 . 0 3 0}$ & 0.38 & 0.112 \\
\hline
\end{tabular}

p<0.05, TSK: Tampa Scale of Kinesiophobia, PCS: Pain Catastrophizing Scale, NPRS: Numerical Pain Rating Scale, FTSTS: Five Times Sit to Stand Test, TUG: Timed Up and Go Test, SCT: Stair Climb Test. 


\section{DISCUSSION}

In this study, the relationship between kinesiophobia and pain catastrophizing with pain, range of motion, muscle strength, and function in knee osteoarthritis patients was investigated. It was determined that there was a relationship between kinesiophobia with pain intensity during activity, active knee flexion angle, FTSTS time, TUG time. Pain catastrophizing was found to be associated with pain intensity at rest, at night and during activity, passive knee extension angle, and active knee flexion angle.

The resting pain intensity of the patients was $5(0-9)$, the pain intensity at night was $6(0-10)$, and the pain intensity during activity was 7 (2-10) in our study. When these values evaluated out of 10 according to NPRS, it is seen that the pain severity of the patients is high. In a study conducted by Aykut Selçuk et al., patients with high kinesiophobia levels also had high pain intensity at resting, at night and during activity (Aykut Selçuk and Karakoyun, 2020). The results of our study show that the pain intensity of patients with knee osteoarthritis are similar to the literature.

In our study, the passive knee flexion angle was 98 (20-115) degrees, and the passive knee extension angle was $1(-5-8)$ degrees. In the study of Steultjens et al., the passive knee flexion and extension angles of the patients were more limited compared to our study (Steultjens et al., 2000). The reason for this situation can be shown as the fact that the patients included in our study have a stage 3 or 4 osteoarthritis level according to the Kellgren-Lawrence Osteoarthritis Classification and that they have limited range of motion at the level for which total knee arthroplasty will be planned. In our study, active knee flexion angles of the patients were 81 (5-109) degrees, and active knee extension angles were 5 (0-10) degrees. In our literature research, no study was found comparing the results of kinesiophobia and active knee range of motions in osteoarthritis patients.

The TUG time in our study was found to be $15.40(8.80-37.90)$ seconds. Since the scores above 14 seconds in the TUG indicate the risk of falling, it was determined that the patients in our study were at risk of falling, similar to the literature (Shumway-Cook et al., 2000). The SCT time of the patients in our study was 30.70 (20.6043.84) seconds, and the FTSTS time was 23.85 (12.40-51.20) seconds.
Kinesiophobia is one of the crucial factors that cause pain and worsening of function after TKA (Perrot et al., 2018). Therefore, it is important to evaluate kinesiophobia and determine its relationship with pain and function in osteoarthritis. A statistically significant relationship was found between kinesiophobia and pain intensity during activity in our study. This result obtained in our study shows that kinesiophobia can be effective not only on function but also on pain. There are conflicting results in the literature on this subject. While there was a relationship between kinesiophobia and pain intensity during activity in the study conducted by Alaca (Alaca, 2019), no relationship was found in the study conducted by Aykut Selçuk et al. (Aykut Selçuk and Karakoyun, 2020). In a systematic review that included 63 studies with a total of 10726 participants, higher levels of kinesiophobia were also found to be associated with higher levels of pain (Luque-Suarez et al., 2019). In our study, it was determined that the active knee flexion angle decreased as the kinesiophobia levels increased. This result shows that kinesiophobia may limit active knee movement in patients with knee osteoarthritis. It was also observed that as the kinesiophobia levels increased, the SCT time and the FTSTS time increased in a correlated way. These results show that kinesiophobia negatively affects the functional status. In the study conducted by Alaca, the functional status assessment was evaluated with the Western Ontario and McMaster Universities Osteoarthritis Index (WOMAC) and a relationship was found between kinesiophobia and WOMAC score (Alaca, 2019). Similarly, in the study conducted by Aykut Selçuk et al., a relationship was found between kinesiophobia and WOMAC score in female patients (Aykut Selçuk and Karakoyun, 2020). The result of these studies shows that the subjective functional status is also affected by kinesiophobia. When the results of these studies and our study are considered together, function worsens as kinesiophobia levels increase.

Pain catastrophizing negatively affects the pain experience by inhibiting pain inhibition in cases of long-term pain (Leeuw et al., 2007). For this reason, pain catastrophizing affects pain and function negatively in osteoarthritis patients. In our study, it was determined that there was a statistically significant relationship between pain catastrophizing and pain intensity at rest, at night, 
and during activity. This result in our study supports the conclusion that pain catastrophizing is the most important psychological factor related to pain (M. J. Sullivan et al., 1995). Similarly, in the study of Odole et al., it was observed that as the pain catastrophizing levels increased, the intensity of pain also increased (Odole et al., 2019). In our study, it was determined that the passive knee extension and active knee flexion angles decreased as the pain catastrophizing levels increased. In our literature research, we did not find any study investigating the correlation between pain catastrophizing and knee range of motion angles. No significant relationship was found between pain catastrophizing and functional tests in our study. In the study conducted by Ong et al., functional status was evaluated with WOMAC and they determined that an increase in pain catastrophizing levels was associated with worsening in function (Ong et al., 2021). The reason for this difference may have arisen from the difference between the level of functional status reported subjectively by patients in WOMAC in the study of Ong et al. and the objective measures of functional tests of our study.

This study had several limitations. One of these limitations was the relatively small sample size. The absence of a healthy control group and a low-level kinesiophobia group was another limitation of the study. Another limitation was not questioning the use and dosage of non-steroidal anti-inflammatory drugs and analgesics before the evaluation. The lack of evaluation of the subjective functional status and quality of life of the patients can also be said as a limitation of the study.

\section{Conclusion}

In conclusion, it was determined that as kinesiophobia increased in patients with knee osteoarthritis, pain, range of motion, muscle strength, and functional status worsened, and the increase in pain catastrophizing was associated with worsening in pain and range of motion. Therefore, it is important to determine the levels of kinesiophobia and pain catastrophizing in patients with knee osteoarthritis. It was concluded that reducing kinesiophobia and pain catastrophizing levels in patients with knee osteoarthritis who had functional limitations would contribute to improve functions.

\section{Conflict of interests}

The authors have no conflict of interests to declare. No financial support was received for this study.

\section{Ethics Statement}

The studies involving human participants were reviewed and approved by the Gazi University, Clinical Research Ethics Committee (Date: 28.06.2021; Decision / Protocol number: 2021/611). Written informed consent to participate in this study was provided by the patients/participants.

\section{REFERENCES}

Abramoff, B., \& Caldera, F. E. (2020). Osteoarthritis: pathology, diagnosis, and treatment options. Medical Clinics, 104(2), 293-311.

Alaca, N. (2019). The relationships between pain beliefs and kinesiophobia and clinical parameters in Turkish patients with chronic knee osteoarthritis: a cross-sectional study. $J$ Pak Med Assoc, 69(6), 823-827.

Ayhan, Y., Karadağ Çaman, Ö., Karahan, S., Kıran, S., Saka, E., Barışkın, E., \& Bilir, N. (2018). A Population-Based Study for the Standardization of the Turkish Version of the Modified Mini Mental State Examination (3MS) and Assessment of Certain Environmental Risk Factors for Dementia: Methodology and Sample Characteristics. Turkish Journal of Psychiatry, 29(4):238-47.

Aykut Selçuk, M., \& Karakoyun, A. (2020). Is There a Relationship Between Kinesiophobia and Physical Activity Level in Patients with Knee Osteoarthritis? Pain Medicine, 21(12), 3458-3469.

Behan, F. P., Maden-Wilkinson, T. M., Pain, M. T., \& Folland, J. P. (2018). Sex differences in muscle morphology of the knee flexors and knee extensors. PloS one, 13(1), e0190903.

Bennell, K., Dobson, F., \& Hinman, R. (2011). Measures of physical performance assessments: Self-Paced walk test (SPWT), stair climb test (SCT), Six-Minute walk test (6MWT), chair stand test (CST), timed up \& go (TUG), sock test, lift and carry test (LCT), and car task. Arthritis care \& research, 63(S11), S350-S370. 
Bijlsma, J. W., Berenbaum, F., \& Lafeber, F. P. (2011). Osteoarthritis: an update with relevance for clinical practice. The Lancet, 377(9783), 2115-2126.

Briggs, M., \& Closs, J. S. (1999). A descriptive study of the use of visual analogue scales and verbal rating scales for the assessment of postoperative pain in orthopedic patients. Journal of pain and symptom management, 18(6), 438-446.

Coderre, T. J., Katz, J., Vaccarino, A. L., \& Melzack, R. (1993). Contribution of central neuroplasticity to pathological pain: review of clinical and experimental evidence. Pain, 52(3), 259-285.

Csuka, M., \& McCarty, D. J. (1985). Simple method for measurement of lower extremity muscle strength. The American journal of medicine, 78(1), 77-81.

DeLoach, L. J., Higgins, M. S., Caplan, A. B., \& Stiff, J. L. (1998). The visual analog scale in the immediate postoperative period: intrasubject variability and correlation with a numeric scale. Anesthesia \& Analgesia, 86(1), 102-106.

Domenech, J., Sanchis-Alfonso, V., López, L., \& Espejo, B. (2013). Influence of kinesiophobia and catastrophizing on pain and disability in anterior knee pain patients. Knee Surgery, Sports Traumatology, Arthroscopy, 21(7), 1562-1568.

Felson, D. T., Lawrence, R. C., Dieppe, P. A., Hirsch, R., Helmick, C. G., Jordan, J. M., . . . Zhang, Y. (2000). Osteoarthritis: new insights. Part 1: the disease and its risk factors. Annals of internal medicine, 133(8), 635-646.

Felson, D. T., Zhang, Y., Hannan, M. T., Naimark, A., Weissman, B. N., Aliabadi, P., \& Levy, D. (1995). The incidence and natural history of knee osteoarthritis in the elderly, the framingham osteoarthritis study. Arthritis \& Rheumatism, 38(10), 1500-1505.

Folstein, M. F., Folstein, S. E., \& McHugh, P. R. (1975). "Mini-mental state": a practical method for grading the cognitive state of patients for the clinician. Journal of psychiatric research, 12(3), 189-198.

Gustavson, A. M., Wolfe, P., Falvey, J. R., Eckhoff, D. G., Toth, M. J., \& StevensLapsley, J. E. (2016). Men and women demonstrate differences in early functional recovery after total knee arthroplasty. Archives of physical medicine and rehabilitation, 97(7), 1154-1162.

Güngen, C., Ertan, T., Eker, E., Yaşar, R., \& Engin, F. (2002). The validity and reliability of the standardized mini mental test in the diagnosis of mild dementia in the Turkish population. Turkish Journal of Psychiatry, 13(4), 273-281.

Hunter, D. J., \& Bierma-Zeinstra, S. M. (2019). Osteoarthritis. The Lancet, 393, 1745-1759.

Jakobsen, T. L., Christensen, M., Christensen, S. S., Olsen, M., \& Bandholm, T. (2010). Reliability of knee joint range of motion and circumference measurements after total knee arthroplasty: does tester experience matter? Physiotherapy Research International, 15(3), 126-134.

James, S. L., Abate, D., Abate, K. H., Abay, S. M., Abbafati, C., Abbasi, N., ... Abdelalim, A. (2018). Global, regional, and national incidence, prevalence, and years lived with disability for 354 diseases and injuries for 195 countries and territories, 1990-2017: a systematic analysis for the Global Burden of Disease Study 2017. The Lancet, 392(10159), 1789-1858.

Jensen, M. P., \& McFarland, C. A. (1993). Increasing the reliability and validity of pain intensity measurement in chronic pain patients. Pain, 55(2), 195-203.

Kacar, C., Gilgil, E., Urhan, S., Arıkan, V., Dündar, Ü., Öksüz, M., Bütün, B. (2005). The prevalence of symptomatic knee and distal interphalangeal joint osteoarthritis in the urban population of Antalya, Turkey. Rheumatology international, 25(3), 201-204.

Kehlet, H., Jensen, T. S., \& Woolf, C. J. (2006). Persistent postsurgical pain: risk factors and prevention. The Lancet, 367(9522), 16181625.

Kori, S. (1990). Kinisophobia: a new view of chronic pain behavior. Pain Manage, 35-43.

Leeuw, M., Goossens, M. E., Linton, S. J., Crombez, G., Boersma, K., \& Vlaeyen, J. W. (2007). The fear-avoidance model of musculoskeletal pain: current state of scientific evidence. Journal of Behavioral Medicine, 30(1), 77-94.

Lespasio, M. J., Piuzzi, N. S., Husni, M. E., Muschler, G. F., Guarino, A., \& Mont, M. A. 
(2017). Knee osteoarthritis: a primer. The Permanente Journal, 21.

Loeser Jr, R. F. (2000). Aging and the etiopathogenesis and treatment of osteoarthritis. Rheumatic Disease Clinics of North America, 26(3), 547-567.

Luque-Suarez, A., Martinez-Calderon, J., \& Falla, D. (2019). Role of kinesiophobia on pain, disability and quality of life in people suffering from chronic musculoskeletal pain: a systematic review. British journal of sports medicine, 53(9), 554-559.

Odole, A., Ekediegwu, E., Ekechukwu, E., \& Uchenwoke, C. (2019). Correlates and predictors of pain intensity and physical function among individuals with chronic knee osteoarthritis in Nigeria. Musculoskeletal Science and Practice, 39, 150-156.

Ong, W. J., Kwan, Y. H., Lim, Z. Y., Thumboo, J., Yeo, S. J., Yeo, W., Leung, Y. Y. (2021). Measurement properties of Pain Catastrophizing Scale in patients with knee osteoarthritis. Clinical rheumatology, 40(1), 295-301.

Perrot, S., Trouvin, A.-P., Rondeau, V., Chartier, I., Arnaud, R., Milon, J.-Y., \& Pouchain, D. (2018). Kinesiophobia and physical therapyrelated pain in musculoskeletal pain: A national multicenter cohort study on patients and their general physicians. Joint Bone Spine, 85(1), 101-107.

Rejeski, W. J., Ettinger Jr, W. H., Schumaker, S., James, P., Burns, R., \& Elam, J. T. (1995). Assessing performance-related disability in patients with knee osteoarthritis. Osteoarthritis and Cartilage, 3(3), 157-167.

Schaubert, K. L., \& Bohannon, R. W. (2005). Reliability and validity of three strength measures obtained from communitydwelling elderly persons. Journal of strength and conditioning research, 19(3), 717.

Shumway-Cook, A., Brauer, S., \& Woollacott, M. (2000). Predicting the probability for falls in community-dwelling older adults using the Timed Up \& Go Test. Physical therapy, 80(9), 896-903.

Steffen, T. M., Hacker, T. A., \& Mollinger, L. (2002). Age-and gender-related test performance in community-dwelling elderly people: Six-Minute Walk Test, Berg Balance
Scale, Timed Up \& Go Test, and gait speeds. Physical therapy, 82(2), 128-137.

Steultjens, M., Dekker, J. v., Van Baar, M., Oostendorp, R., \& Bijlsma, J. (2000). Range of joint motion and disability in patients with osteoarthritis of the knee or hip. Rheumatology, 39(9), 955-961.

Streiner, D. L., Norman, G. R., \& Cairney, J. (2015). Health measurement scales: a practical guide to their development and use: Oxford University Press, USA.

Sullivan, M., Tanzer, M., Stanish, W., Fallaha, M., Keefe, F. J., Simmonds, M., \& Dunbar, M. (2009). Psychological determinants of problematic outcomes following total knee arthroplasty. Pain, 143(1-2), 123-129.

Sullivan, M. J. (2009). The pain catastrophizing scale: user manual. Montreal: McGill University, 1-36.

Sullivan, M. J., Bishop, S. R., \& Pivik, J. (1995). The pain catastrophizing scale: development and validation. Psychological assessment, 7(4), 524.

Tiedemann, A., Shimada, H., Sherrington, C., Murray, S., \& Lord, S. (2008). The comparative ability of eight functional mobility tests for predicting falls in community-dwelling older people. Age and ageing, 37(4), 430-435.

Ugurlu, M., Karakas Ugurlu, G., Erten, S., \& Caykoylu, A. (2017). Validity of Turkish form of Pain Catastrophizing Scale and modeling of the relationship between painrelated disability with pain intensity, cognitive, and emotional factors. Psychiatry and Clinical Psychopharmacology, 27(2), 189-196.

Vlaeyen, J. W., Kole-Snijders, A. M., Boeren, R. G., \& Van Eek, H. (1995). Fear of movement/(re) injury in chronic low back pain and its relation to behavioral performance. Pain, 62(3), 363-372.

Vlaeyen, J. W., \& Linton, S. J. (2000). Fearavoidance and its consequences in chronic musculoskeletal pain: a state of the art. Pain, 85(3), 317-332.

Watkins, M. A., Riddle, D. L., Lamb, R. L., \& Personius, W. J. (1991). Reliability of goniometric measurements and visual estimates of knee range of motion obtained in a clinical setting. Physical therapy, 71(2), 90-96. 
Woolf, A. D., \& Pfleger, B. (2003). Burden of major musculoskeletal conditions. Bulletin of the world health organization, 81, 646-656.

Woolf, C. J., \& Salter, M. W. (2000). Neuronal plasticity: increasing the gain in pain. science, 288(5472), 1765-1768.

Yilmaz, Ö. T., Yakut, Y., Uygur, F., \& Uluğ, N. (2011). Turkish version of Tampa Kinesiophobia Scale and test-retest reliability. Physiotherapy Rehabilitation, 22(1), 44-49.

Zhang, Y., \& Jordan, J. M. (2010). Epidemiology of osteoarthritis. Clinics in geriatric medicine, 26(3), 355-369.

How to cite this article: Gür, O., Başar, S., Esen, E., Ataoğlu, M.B. and Turanlı, S. (2021 The Relationship of Kinesiophobia and Pain Catastrophizing with Pain, Range of Motion, Muscle Strength and Function in Osteoarthritis. Int $J$ Disabil SportsHealth Sci;4(2):130-139.https://doi.org/10.3343 /ijdshs. 980343 